LOUIS E. LOEB

\title{
ON A HEADY ATTEMPT TO BEFIEND CAUSAL THEORIES OF KNOWLEDGE*
}

\author{
(Received 6 March, 1975)
}

In 1967, Alvin Goldman proposed that a person $X$ knows that $p$ only if the fact that $p$ is causally connected with $X$ 's belief that $p \cdot{ }^{1}$ In the same journal issue, Brian Skyrms produced an alleged counterexample:

Let us suppose that ' $\mathrm{Z}$ 's head has been cut off' (' $\mathrm{Fa}$ ') is causally sufficient for ' $Z$ is dead' (' $G a$ '). $X$ is walking along the street and notices $Z$ lying in the gutter, with head severed from body. On the basis of his background knowledge, he arrives at the belief that $Z$ is dead. Sure enough, $Z$ is dead. Now suppose that the sequence of events leading to this pretty scene were as follows: $Z$ was lying in the street, drunk and motionless. Then he died of a heart attack. Finally, a fiend chanced upon the scene and, seeing a man lying in the gutter (but not knowing he was dead) he whacked off his head. Notice that, in the ordinary sense of 'cause', Z's losing his head was not the cause of death. In fact, it is not even causally connected with his death. Nevertheless, this would not prevent us from granting that $X$ knew that $Z$ was dead. ${ }^{2}$

Recently, this case has won wide acceptance as a counterexample to Goldman's causal theory of knowledge: from Robert Ackermann, Gilbert Harman, and Marshall Swain. ${ }^{3}$ Of these, only Swain believes that Goldman's theory can be modified to accommodate the Skyrms case. I argue below that the Skyrms case is not a counterexample to Goldman's original theory. To see this, we must attend to the difference between a person's death and a person's being dead, and to distinctions related to that between causation and causal overdetermination. 4

Some events, conditions, and states of affairs are causally overdetermined. For example, a short circuit starts a fire in a house's kitchen. At the same time, a cigarette ash starts a fire in the master bedroom. Either fire alone would have spread and destroyed the house completely in one hour. In one hour the house is completely destroyed - its destruction is causally overdetermined. In these circumstances, the short circuit and the cigarette ash are causally overdetermining factors, or causal overdeterminants, of the house's destruction. There is some reluctance to say of these factors that they are, strictly speaking, causes of the house's 
destruction. (Perhaps this is because neither the short circuit nor the cigarette ash was necessary for the house's destruction, even in the circumstances.) For this reason, I use the term 'cause' in a strict sense such that causal overdeterminants are not causes; and I use the term 'C-condition' to embrace both strict causes and causal overdeterminants. ${ }^{5}$ Others use 'cause' more liberally in a way which coincides with my use of 'C-condition'.

Goldman holds that $X$ knows that $p$ only if the fact that $p$ is causally connected with $X$ 's belief that $p$. He says that two events, conditions, or states of affairs are causally connected just in case either one is a cause of the other or both have a cause in common. ${ }^{6}$ Should 'cause' in the account of 'causal connections' be interpreted narrowly to coincide with 'cause' in my strict sense, or liberally to coincide with 'C-condition'? It is clear that Goldman uses 'cause' liberally. In a footnote, he considers "... a table top that is supported by four legs. When a fifth leg is inserted flush beneath the table top, it too becomes a cause of the table top's not falling." " In my terminology, the fifth leg is a causal overdeterminant and a C-condition, but not a cause, of the table top's not falling. We now know how to interpret Goldman: the fact that $p$ is causally connected with $X$ 's belief that $p$ just in case either the fact that $p$ is a C-condition of $X$ 's belief that $p$, or both have a C-condition in common.

In the Skyrms case, $X$ sees that $Z$ 's head is severed, and infers that " $Z$ is dead" or " $Z$ was dead". Presumably, $X$ does not infer that $Z$ was dead ten years before. If $X$ is circumspect, he will note that ' $Z$ 's head has been cut off by $t$ ' is causally sufficient for ' $Z$ is dead at $t+\mathrm{e}$ ', where epsilon represents some slight time lag. Suppose $X$ encounters the decapitated body at time $t_{4}$. Then $X$ knows that $Z$ 's head is severed by $t_{4}$ and $X$ infers that $Z$ is dead at $t_{4+\mathrm{e}}(p)$. Is the fact that $p$ causally connected with $X$ 's belief that $p$ ? The sequence of events can be diagrammed as follows (arrows are to be read: "... is a C-condition for..."):

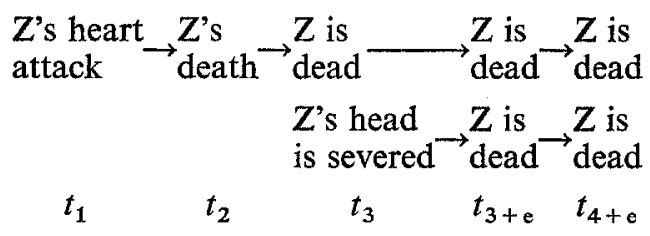

$Z$ 's death is not causally overdetermined; the heart attack in particular 
is a (strict) cause and not a causal overdeterminant of $Z$ 's death. Similarly, $Z$ 's being dead at each moment from $t_{2}$ up until $t_{3+\mathrm{e}}$ is not causally overdetermined; the heart attack is therefore a (strict) cause of $Z$ 's being dead at each of these moments. These features of the Skyrms case easily obscure the fact that at $t_{3+e}$ and thereafter $Z$ 's being dead is causally overdetermined; the heart attack and the severing are causal overdeterminants (and hence C-conditions but not strict causes) of $Z$ 's being dead at $t_{3+\mathrm{e}}$ and thereafter (and at $t_{4+\mathrm{e}}$ in particular).

If the severing had by chance occurred at $t_{2-\mathrm{e}}, Z$ 's death would have been causally overdetermined, and it would be obvious that $Z$ 's being dead at any particular time is causally overdetermined. The fact that the severing comes after death makes no difference. In this respect, the severing is like the fifth leg inserted flush beneath a table top already supported by four legs. The fifth leg is a causal overdeterminant of the table top's not falling at times after the fifth leg has been inserted. The severing is a causal overdeterminant of $Z$ 's being dead at times after the severing has occurred.

I have pointed out that the severing is a causal overdeterminant, and hence a C-condition, of the fact that $p, Z$ 's being dead at $t_{4+\mathrm{e}}$. The severing is clearly a cause, and hence a C-condition, of $X$ 's belief that $p$ : the severing is a cause of $Z$ 's head's being separated from his body after $t_{3} ; X$ sees head and body so separated at $t_{4}$, and infers that $p$, that $Z$ is dead at $t_{4+\mathrm{e}}$ (both seeing and inference are assumed to be causal processes). The fact that $p$ and $X$ 's belief that $p$ are therefore causally connected; they have a C-condition in common, the severing. ${ }^{8}$

Previous discussions of the Skyrms case overlook this causal connection because they confuse the fact that $p$, a state of affairs at $t_{4+e}$, with $Z$ 's death, an event at $t_{2}$. Thus we have:

(Skyrms) ...Z's losing his head ... is not even causally connected with his death...this would not preclude us from granting that $\mathrm{X}$ knew that $Z$ was dead.

(Ackermann)... a knows that Brown is dead. but it happens that there is no causal chain connecting death and decapitation.

(Harman) ... you come to know that he is dead... having his head cut off did not cause Omar's death, since he was already dead.

(Swain) Even though you know that he is dead... there is no event in the causal chain leading to your belief that his head has been severed from his body which is also in fact causally responsible for his death .... ${ }^{9}$

All such points are irrelevant since there is a causal connection, via the 
severing, between $X$ 's belief that $Z$ is dead at $t_{4+\mathrm{e}}$ and $Z$ 's being dead at $t_{4+\mathrm{e}}$. The Skyrms case is not a counterexample to Goldman's causal theory of knowledge.

The quotations above might suggest that a minor modification of the Skyrms case will yield a counterexample after all. Is not the real point that $X$ knows that $Z$ died, even though $Z$ 's death, or dying, is not causally connected with $X$ 's belief that $Z$ died? If $X$ infers that $Z$ died from his (knowledge constituting) belief that $Z$ is dead at $t_{4+\mathrm{e}}$, we want to say that $X$ knows that $Z$ died. Goldman's analysis requires that $Z$ 's dying is causally connected with $X$ 's belief that $Z$ died. Is this condition satisfied?

In the article introducing his causal theory of knowledge, Goldman stated the following principle:

(GP) if $q$ is logically related to $p$, and if $p$ is a cause of $z$, then $q$ is a cause of $z_{.}{ }^{10}$

GP (Goldman's principle) has been stated carelessly. If $p$ and $q$ are logically related, they are presumably statements or propositions. But statements and propositions are not causes. Goldman should have said:

$\left(\mathrm{GP}^{\prime}\right)$ if $q$ is logically related to $p$, and if the fact that $p$ is a cause of $z$, then the fact that $q$ is a cause of $z$.

Substituting "is causally connected with" for both occurrences of "is a cause of" yields a modest generalization of $\mathrm{GP}^{\prime}$ :

$\left(\mathrm{GGP}^{\prime}\right)$ if $q$ is logically related to $p$, and if the fact that $p$ is causally connected with $z$, then the fact that $q$ is causally connected with $z$.

GGP' is easily applied to the modified Skyrms example. In the special case where $z$ is $X$ 's belief that $q, \mathrm{GGP}^{\prime}$ reads:

if $q$ is logically related to $p$, and if the fact that $p$ is causally connected with $X$ 's belief that $q$, then the fact that $q$ is causally connected with $X$ 's belief that $q$. 
" $Z$ is dead at $t_{4+c} "(p)$ is logically related to " $Z$ died" $(q)$ because the former entails the latter. Given (as I have shown above) that the fact that $p$ is causally connected with $X$ 's belief that $p$, and given that $X$ 's belief that $p$ is a cause of $X$ 's belief that $q$ (by inference), the fact that $p$ is causally connected with $X$ 's belief that $q$. (The fact that $p$ and $X$ 's belief that $q$ have a C-condition in common, the severing). It follows by GGP' that the fact that $q$ is causally connected with $X$ 's belief that $q$. That is, $Z$ 's dying is causally connected with $X$ 's belief that $Z$ died in Goldman's sense of "causally connected". ${ }^{11}$ The modified Skyrms case would be a counterexample to Goldman's original analysis without the modest generalization of $\mathrm{GP}^{\prime}$. I am certain that Skyrms and those following him in the use of the mad fiend case have not wanted to show the conditions of Goldman's analysis too strong in this trivially reparable respect. In any case, the modified Skyrms case is not a counterexample to Goldman's analysis as revised above.

One might object, on various grounds, to $\mathrm{GP}^{\prime}$ (and hence to $\mathrm{GGP}^{\prime}$ ) itself. It is my own view that there are serious problems both for these principles, and for other features of Goldman's causal theory of knowledge. I do not want to defend that theory. I do want correctly to locate the serious difficulties for that theory. The original Skyrms case is the most visible and repeated objection to Goldman's analysis; it has been treated as a simple and devastating counterexample which has cleared the way for ready dismissal of the theory. In fact, it is no counterexample at all. The modified Skyrms case fares no better. I hope I have contributed to undermining the role of these alleged counterexamples in philosophical discussion.

The University of Michigan

\section{NOTES}

* I am grateful to Alvin Goldman and David Lewis for some useful suggestions. 1 Alvin I. Goldman, 'A Causal Theory of Knowing', The Journal of Philosophy LXIV, 12 (June 22, 1967), 357-372, pp. 358, 369.

2 Brian Skyrms, "The Explication of 'X Knows that $p$ ", The Journal of Philosophy LXIV, 12 (June 22, 1967), 373-389, pp. 285-6. Also cf. footnote 17, p. 382.

3 Cf. Robert J. Ackermann, Belief and Knowledge (Garden City, New York, Anchor Books, 1972), p. 96; Gilbert Harman, Thought (Princeton, New Jersey, Princeton University Press, 1973), pp. 134-5; and Marshall Swain, 'Knowledge, Causality, and 
Justification', The Journal of Philosophy LXIX, 11 (June 1, 1972), 291-300, pp. 295-6. 4 After writing this paper, I discovered that D. Goldstick has proposed essentially the same dissolution of the Skyrms case, compressed into a single paragraph of his 'A Contribution Towards the Development of the Causal Theory of Knowledge', The Australasian Journal of Philosophy 50, 3 (December, 1972), 238-248, pp. 241-2. Goldstick's treatment of the case, however, requires that we countenance the existence of disjunctive events which are causes. This aside, $I$ believe the present more extended discussion of the case is warranted given that two recent books (see footnote 3) produce Skyrms' example as a counterexample, and a decisive one, to Goldman's causal theory of knowledge.

5 Elsewhere, I have provided analyses of the intuitive concepts of 'cause', 'causal overdeterminant', and 'C-condition'. Matters are complicated because causally overdetermined events typically have some strict causes as well as some causal overdeterminants. But this does not affect any points in this paper. See my 'Causal Theories and Causal Overdetermination', The Journal of Philosophy LXXI, 15 (Sept. 5, 1974), $525-44$.

${ }^{6} C f$. Goldman, op. cit., p. 364.

7 Ibid., p. 362.

8 The fact that $p$ and $X$ 's belief that $p$ are also causally connected by virtue of the existence of common $\mathrm{C}$-conditions other than the severing. For example, they are causally connected via the head's being separated from the body at $t_{4}$; this state is a cause of $X$ 's belief that $p$, and a causal overdeterminant of the fact that $p$.

${ }^{9}$ Skyrms, op. cit., p. 386; Ackermann, op. cit., p. 96; Harman, op. cit., p. 134; and Swain, op. cit., p. 296 (italics variously deleted and added).

10 Wherever I have written ' $q$ ' and ' $p$ ', Goldman reads ' $x$ ' and ' $y$ ', respectively. Goldman, op. cit., p. 368.

11 My application of GGP' is precisely analogous to Goldman's application of GP. Cf. ibid., pp. 368-9. 